VOL. 45 (1992) [399-413]

\title{
HAAR MEASURE AND COMPACT RIGHT TOPOLOGICAL GROUPS
}

\author{
Paul Milnes
}

The consideration of compact right topological groups goes back at least to a paper of Ellis in 1958, where it is shown that a flow is distal if and only if the enveloping semigroup of the flow is such a group (now called the Ellis group of the distal flow). Later Ellis, and also Namioka, proved that a compact right topological group admits a left invariant probability measure. As well, Namioka proved that there is a strong structure theorem for compact right topological groups. More recently, John Pym and the author strengthened this structure theorem enough to be able to establish the existence of Haar measure on a compact right topological group, a probability measure that is invariant under all continuous left and right translations, and is unique as such. Examples of compact right topological groups have been considered earlier. In the present paper, we give concrete representations of several Ellis groups coming from low dimensional nilpotent Lie groups. We study these compact right topological groups, and two others, in some detail, paying attention in particular to the structure theorem and Haar measure, and to the question: is Haar measure uniquely determined by left invariance alone? (It is uniquely determined by right invariance alone.) To assist in answering this question, we develop some sufficient conditions for a positive answer. We suspect that one of the examples, a compact right topological group coming from the Euclidean group of the plane, does not satisfy these conditions; we don't know if the question has a positive answer for this group.

\section{Compact Right Topological Groups}

A compact right topological group $(G, \tau)$ is a group that is also a compact, Hausdorff, topological space with topology $\tau$ for which the right multiplications $x \mapsto x y$, $G \rightarrow G$ are all continuous. The groups that we consider here also have the property that the topological centre

$$
\Lambda(G):=\{y \in G \mid x \mapsto y x, \quad G \rightarrow G \text { is continuous }\}
$$

is dense in $G$. This property characterises the compact right topological groups that arise as the Ellis groups of distal flows. There is a powerful structure theorem for such

Received 9 May 1991

This research was supported in part by NSERC grant A7857

Copyright Clearance Centre, Inc. Serial-fee code: 0004-9729/92 \$A2.00+0.00. 
groups $[13,3,11]$. A very simple case of it suffices for the examples we want to consider here. We will assume that

(*) $G$ has a smallest compact normal subgroup $(L, \tau)$ with the properties that the quotient group $(G / L, \tau)$ is a topological group and the restricted multiplication mapping

$$
(x, y) \mapsto x y, G \times L \rightarrow G
$$

is (jointly) continuous.

We point out that the continuity condition implies that $L$ (as well as $G / L$ ) is a topological group, and that we are using $\tau$ also to denote subspace and quotient space topologies coming from $\tau$. An explicit expression for $L$ is

$$
L=\bigcap\left\{U^{-1} U U^{-1} U \mid U \text { is a neighbourhood of the identity of } G\right\} .
$$

See $[13,3,11]$ for all these matters.

A consequence of the structure theorem (in the present setting and in general) is that $G$ has a Haar measure $\mu$, which we regard as a linear functional on $\mathcal{C}(G)$, the $C^{*}$-algebra of continuous $\mathbb{C}$-valued functions on $G$. Now $\mu$ is

(i) a probability measure on $G$;

(ii) right invariant, that is, $\mu\left(R_{x} f\right)=\mu(f)$ for all $x \in G$ and $f \in \mathcal{C}(G)$; and

(iii) left invariant, that is, $\mu\left(L_{x} f\right)=\mu(f)$ for all $x \in \Lambda(G)$ and $f \in \mathcal{C}(G)$.

(Here $R_{x} f(y)=f(y x)$ and $L_{x} f(y)=f(x y)$, as usual.) The probability measure $\mu$ is uniquely determined by (ii) [11] and is constructed from the Haar measures $\mu_{1}$ and $\mu_{2}$ on the compact topological groups $L$ and $G / L$. (We remind the reader that Haar measure on a compact topological group is both right and left invariant, is uniquely determined by either of these properties, and is uniquely determined even by invariance under left, or right, translations by all members of a dense subset of the group [6, Chapter IV].) Haar measure $\mu$ on $G$ is constructed as follows. For

$$
f \in \mathcal{C}_{L}(G):=\{f \in \mathcal{C}(G) \mid f \text { is constant on the cosets } x L \text { of } G\} \cong \mathcal{C}(G / L),
$$

$\mu(f)$ is defined to be $\mu_{2}(f)$. For $f \in \mathcal{C}(G)$, we define first

$$
\bar{f}(x):=\int_{L} f(x y) d \mu_{1}(y)=\int_{L} \dot{L}_{x} f d \mu_{1} .
$$

The continuity condition in $(*)$ assures us that $\left.L_{x} f\right|_{L} \in \mathcal{C}(L)$ and that $\bar{f} \in \mathcal{C}(G)$; in fact, $\bar{f} \in \mathcal{C}_{L}(G)$, by the invariance of $\mu_{1}$. So, we define

$$
\mu(f):=\mu(f)=\mu_{2}(f) .
$$


Since $f=\bar{f}$ for $f \in \mathcal{C}_{L}(G)$, this definition coincides with the first one given for such functions.

Note particularly that the Haar measure on a compact right topological group $G$ is not necessarily uniquely determined by left invariance alone even if $\Lambda(G)$ contains an (algebraically) isomorphic copy of $\mathbb{Z}$ that is dense in $G$ [2].

The theorem which follows gives a setting where Haar measure is the unique left invariant probability measure on a compact right topological group. We need a strong (joint) continuity hypothesis for it, (i) in the next lemma.

LEMMA 1. For a compact right topological group $G$ satisfying (*), the following assertions are equivalent.

(i) $(x, y) \mapsto x^{-1} y x, G \times L \rightarrow L$ is continuous.

(ii) $(x, y) \mapsto y x, G \times L \rightarrow G$ is continuous.

Proof: Recall that $L$ is normal in $G$, and set

$$
B:=\{(x, x y) \mid x \in G, \quad y \in L\}=\{(x, y x) \mid x \in G, y \in L\} \subset G^{2} .
$$

If (i) holds, it follows that $u:(x, y) \mapsto\left(x, x^{-1} y x\right), G \times L \rightarrow G \times L$ is continuous; since $u$ is $1-1$, it is a homeomorphism. The continuity hypothesis of (*), yields the same conclusion about $v:(x, y) \mapsto(x, x y), G \times L \rightarrow B$. Then $v \circ u:(x, y) \mapsto(x, y x)$, $G \times L \rightarrow B$ is continuous, which gives (ii). Conversely, if (ii) holds, then $v \circ u$ is continuous, as is $u=v^{-1} \circ(v \circ u)$, which gives (i).

THEOREM 2. Suppose that $G$ is a compact right topological group satisfying (*) and that $\Lambda(G) \supset L$. Suppose as well that (i) (equivalently, (ii)) of Lemma 1 is satisfied. Then Haar measure $\mu$ on $G$ is uniquely determined by left invariance.

Proof: Suppose that $\mu^{\prime}$ is a left invariant probability measure on $G, \mu^{\prime}\left(L_{y} f\right)=$ $\mu^{\prime}(f)$ for all $f \in \mathcal{C}(G)$ and all $y \in \Lambda(G)$. Since $\Lambda(G)$ is dense in $G$, we see immediately that $\mu^{\prime}$ agrees with $\mu$ on $\mathcal{C}_{L}(G)$, because left invariance forces the restriction of $\mu^{\prime}$ to

$$
\mathcal{C}_{L}(G)=\{f \in \mathcal{C}(G) \mid f \text { is constant on each coset } x L\} \cong \mathcal{C}(G / L)
$$

to be just integration with respect to Haar measure $\mu_{2}$ on $G / L$, which is the definition of $\mu$ on $\mathcal{C}_{L}(G)$. Let $f \in \mathcal{C}(G)$. Bearing in mind the hypothesis $L \subset \Lambda(G)$, we then have

$$
\begin{aligned}
\mu^{\prime}(f) & =\int_{L} \mu^{\prime}(f) d \mu_{1}(y)=\int_{L} \mu^{\prime}\left(L_{y} f\right) d \mu_{1}(y)=\int_{L} \int_{G} f(y x) d \mu^{\prime}(x) d \mu_{1}(y) \\
& =\int_{G} \int_{L} f\left(x\left(x^{-1} y x\right)\right) d \mu_{1}(y) d \mu^{\prime}(x)=\int_{G} \int_{L} f(x y) d \mu_{1}(y) d \mu^{\prime}(x) \\
& =\int_{G} \bar{f}(x) d \mu^{\prime}(x)=\mu^{\prime}(\bar{f})=\mu(f)=\mu(f)
\end{aligned}
$$


the fifth equality holding because the continuous automorphism $y \mapsto x^{-1} y x$ of $L$ leaves Haar measure $\mu_{1}$ on $L$ invariant, and the eighth one holding because $\bar{f} \in \mathcal{C}_{L}(G)$ and $\mu^{\prime}=\mu$ on $\mathcal{C}_{L}(G)$.

The assumption (*) says that we are dealing with group extensions. We need to establish notation for Schreier's analysis of such extensions. Suppose that $G_{1}$ and $G_{2}$ are groups, the identity of each of them being denoted by $e$. Suppose that there is a homomorphism of $G_{2}$ into the automorphism group of $G_{1}$, that is, for every $t \in G_{2}$, there is an automorphism $s \mapsto t(s)$ of $G_{1}$ (acting on the left). Suppose also that there is a function $\left(t^{\prime}, t\right) \mapsto\left[t^{\prime}, t\right]$ from $G_{2} \times G_{2} \rightarrow G_{1}$, so that the following conditions are satisfied:

$$
\begin{gathered}
e(s)=s \text { and }[t, e]=[e, t]=e \text { for } s \in G_{1}, t \in G_{2}, \text { and also } \\
{\left[t, t^{\prime}\right] t t^{\prime}\left(s^{\prime \prime}\right)=t\left(t^{\prime}\left(s^{\prime \prime}\right)\right)\left[t, t^{\prime}\right] \text { and }\left[t, t^{\prime}\right]\left[t t^{\prime}, t^{\prime \prime}\right]=t\left(\left[t^{\prime}, t^{\prime \prime}\right]\right)\left[t, t^{\prime} t^{\prime \prime}\right]} \\
\text { for } t, t^{\prime}, t^{\prime \prime} \in G_{2} \text { and } s s^{\prime}, s^{\prime \prime} \in G_{1} .
\end{gathered}
$$

Note that the function sending $t$ to the automorphism $s \mapsto t(s)$ of $G_{2}$ is not necessarily a homomorphism. However, the hypotheses do ensure that the formula

$$
\left(s^{\prime}, t^{\prime}\right)(s, t)=\left(s^{\prime} t^{\prime}(s)\left[t^{\prime}, t\right], t^{\prime} t\right)
$$

defines a group operation on the set $G=G_{1} \times G_{2}$, that $G_{1} \times\{e\}$ is a normal subgroup isomorphic to $G_{1}$, and that $G / G_{1} \cong G_{2}$. We say that $G$ is an extension of $G_{1}$ by $G_{2}$. Further, if $G$ is a group with a normal subgroup $G_{1}$ and $G_{2}:=G / G_{1}$, then one can find functions satisfying the conditions above, so that $G$ is canonically isomorphic (algebraically) to $G_{1} \times G_{2}$ with group operation (1).

Because of the asymmetry of continuity in the definition of compact right topological group, we also need the notation for the analogous situation, where $G=G_{1} \times G_{2}$ is an extension of $G_{2}$ by $G_{1}$, that is, $G_{2}$ is a normal subgroup of $G$ and $G_{1} \cong G / G_{2}$. In this case, we have automorphisms $t \mapsto(t) s$ of $G_{2}$ (acting on the right) and the multiplication formula is

$$
\left(s^{\prime}, t^{\prime}\right)(s, t)=\left(s^{\prime} s,\left[s^{\prime}, s\right]\left(t^{\prime}\right) s t\right)
$$

The reader will see how the two forms are needed to deal with the examples. In another paper, we will use the two forms alternately to generalise a construction of Ruppert [16]. A situation in which left and right notations can be used at the same time is that of Zappa products $[17,3]$, where a group $G$ has subgroups $G_{1}$ and $G_{2}$ with

$$
G=G_{1} G_{2}=\left\{s t \mid s \in G_{1}, t \in G_{2}\right\} \text { and } G_{1} \cap G_{2}=\{e\}
$$


Then $G$ is (algebraically) isomorphic to $G_{1} \times G_{2}$ with operation

$$
\left(s^{\prime}, t^{\prime}\right)(s, t)=\left(s^{\prime} t^{\prime}(s),\left(t^{\prime}\right) s t\right)
$$

here the functions $s \mapsto t^{\prime}(s)=\mathcal{L}_{t^{\prime}}(s)$ and $t^{\prime} \mapsto\left(t^{\prime}\right) s=\left(t^{\prime}\right), \mathcal{R}$ are not necessarily automorphisms or even endomorphisms of $G_{1}$ and $G_{2}$, respectively; however, the maps

$$
t^{\prime} \mapsto \mathcal{L}_{t^{\prime}}, G_{2} \rightarrow G_{1}^{G_{1}} \text { and } s \mapsto, \mathcal{R}, G_{1} \rightarrow G_{2}^{G_{2}}
$$

are homomorphisms (the group operation in $G_{1}^{G_{1}}$ and $G_{2}^{G_{2}}$ being composition of functions). The conditions that make the Zappa operation associative are

$$
\mathcal{L}_{t^{\prime}}\left(s^{\prime} s\right)=\mathcal{L}_{t^{\prime}}\left(s^{\prime}\right)\left[\mathcal{L}_{\left(\left(t^{\prime}\right),,^{\prime}\right)}(s)\right] \text { and }\left(t^{\prime} t\right), \mathcal{R}=\left[\left(t^{\prime}\right)_{\left(\mathcal{L}_{t}(s)\right)} \mathcal{R}\right](t), \mathcal{R}
$$

Corollary 3. Suppose that $G_{1}$ and $G_{2}$ are compact topological groups, and that the product space $G:=G_{1} \times G_{2}$ has multiplication (1), or (2), which makes it a compact right topological group. With (1), Haar measure $\mu$ on $G$ is always uniquely determined by left invariance. With (2), $L$ is (isomorphic to) a subgroup of $G_{2}, L \subset$ $\{e\} \times G_{2}$, and $\mu$ is uniquely determined by left invariance if $L \subset \Lambda(G)$ and the action

$$
(s, t) \mapsto(t) s, G_{1} \times L \rightarrow L
$$

is continuous.

Proof: In setting (1), we have containments $L \subset G_{1} \times\{e\} \subset \Lambda(G)$, which follow from the form of $L$ given after $(*)$ and the multiplication formula (1). The map (ii) of Lemma $1,(x, y) \mapsto y x, G \times L \rightarrow G$ is then just

$$
\left(\left(s^{\prime}, t^{\prime}\right),(s, e)\right) \mapsto(s, e)\left(s^{\prime}, t^{\prime}\right)=\left(s s^{\prime}, t\right)
$$

which is continuous, since $G_{1}$ is a topological group.

In setting (2), $L \subset\{e\} \times G_{2}$ and the continuity hypothesis ensures that

$$
\left(\left(s^{\prime}, t^{\prime}\right),(e, t)\right) \mapsto(e, t)\left(s^{\prime}, t^{\prime}\right)=\left(s^{\prime},(t) s^{\prime} t^{\prime}\right), G \times L \rightarrow G
$$

is continuous.

Thus, in both settings, the hypotheses of the theorem have been satisfied, and we get the desired conclusion. 


\section{EXAMPLES}

We present three examples, identifying (in most cases) the subgroups $\Lambda(G)$ and $L$, and Haar measure, among other things. Some aspects of the first example have been discussed in [7], of the second and third in $[13,3,8]$, where other examples of compact right topological groups are given.

Let us indicate how we find these compact right topological groups. We start with a minimal distal flow $(S, X)$ and identify $S$ (sometimes a homomorphic image of $S$ ) in the natural way with a subset of $X^{X}$; then the enveloping semigroup $G:=S^{-} \subset X^{X}$ is a compact right topological group with $S \subset \Lambda(G)$ dense in $G$ (closure, density, et cetera being in the product topology) if and only if $(S, X)$ is distal. One way of getting such a flow begins with a locally compact topological group $S$ and subgroup $H \subset S$ such that the quotient space $X:=S / H$ is compact. Then $(S, X)$ with action

$$
(s, t H) \mapsto s t H, S \times X \rightarrow X
$$

is always a minimal flow; we don't bother to check specifically for distality in our examples (although this is not hard to do), because the enveloping semigroups turn out to be groups, which implies that the flows are distal. See [5, Chapter 6] (or [3, 1.6.18(e)]) for all this. One task that is always a part of these deliberations is to identify $G$, which starts out as $S^{-}$, a usually "small" closed subset of the "large" product space $X^{X}$, in some more reasonable way. We are unable to complete this task for Example 2 , and the problem seems even worse for an example given in Remark 2(iv).

For ease of reference, we quote a consequence of Pontrjagin duality for the almost periodic compactification $\mathfrak{G}^{\mathcal{A P}}$ of a locally compact abelian group $\mathfrak{G}$, which we identify, as a set, with its canonical image in $\mathfrak{G}^{\mathcal{A P}}[\mathbf{1}]$, or $[3,4.3 .18]$.

THEOREM 4. Let $\mathfrak{G}$ be a locally compact abelian group with dual group $\widehat{\mathfrak{G}}=\mathfrak{G}$. Then $\mathfrak{G}^{\mathcal{A P}}=\left((\widehat{\mathfrak{G}})_{d}\right)$, and the members of $\mathfrak{G}^{\mathcal{A P}}$ that are continuous on $\widehat{\mathfrak{G}}$ are precisely the ones in $\mathfrak{G}$.

EXAMPLE 1. Let $S$ be $\mathbb{R}^{3}$ with multiplication

$$
\left(x_{1}^{\prime}, x_{2}^{\prime}, x_{3}^{\prime}\right)\left(x_{1}, x_{2}, x_{3}\right)=\left(x_{1}^{\prime}+x_{1}+x_{3}^{\prime} x_{2}, x_{2}^{\prime}+x_{2}, x_{3}^{\prime}+x_{3}\right)
$$

This is the (connected and simply connected) nilpotent Lie group of dimension 3 and may be viewed as the group of (real) $3 \times 3$ matrices with ones on the diagonal and zeroes below the diagonal. Let $H$ be the non-normal subgroup $\left\{\left(x_{1}, x_{2}, x_{3}\right) \mid x_{1}, x_{2}, x_{3} \in \mathbb{Z}\right\}$. We deal with the flow $(S, S / H)$ as indicated above. (In [5, pp.53-4], Ellis gives a quite different discussion of this flow.) Now, each coset $\left(x_{1}, x_{2}, x_{3}\right) H \in S / H$ contains a 
unique member $\left(x_{1}^{\prime}, x_{2}^{\prime}, x_{3}^{\prime}\right) \in X:=[0,1)^{3} ;\left(x_{1}^{\prime}, x_{2}^{\prime}, x_{3}^{\prime}\right)$ satisfies

$$
\begin{gathered}
\left(x_{1}^{\prime}, x_{2}^{\prime}, x_{3}^{\prime}\right)^{-1}\left(x_{1}, x_{2}, x_{3}\right)=\left(-x_{1}^{\prime}+x_{2}^{\prime} x_{3}^{\prime},-x_{2}^{\prime},-x_{3}^{\prime}\right)\left(x_{1}, x_{2}, x_{3}\right) \\
=\left(-x_{1}^{\prime}+x_{2}^{\prime} x_{3}^{\prime}+x_{1}-x_{3}^{\prime} x_{2},-x_{2}^{\prime}+x_{2},-x_{3}^{\prime}+x_{3}\right) \in H,
\end{gathered}
$$

that is, $x_{3}^{\prime}=x_{3}(\bmod ) 1, x_{2}^{\prime}=x_{2}(\bmod 1)$, and

$x_{1}^{\prime}=\left(x_{1}-x_{3}(\bmod 1) x_{2}+x_{2}(\bmod 1) x_{3}(\bmod 1)\right) \bmod d 1=\left(x_{1}-x_{3}\left[x_{2}\right]\right)(\bmod 1)$,

where $\left[x_{2}\right]$ is the integral part of $x_{2}$, as usual. To get the topology of $X$ right (that is, so that $X$ is homeomorphic to the quotient space $S / H$ and the quotient map

$$
S \rightarrow S / H \cong X
$$

is continuous), it is convenient to identify $X$ with $\mathbb{T} \times[0,1) \times \mathbb{T}$ via

$$
\left(c_{1}, c_{2}, c_{3}\right) \cong\left(e^{2 \pi i c_{1}}, c_{2}, e^{2 \pi i c_{3}}\right) \text {. }
$$

Then the quotient map $S \rightarrow X$ is given by

$$
\left(x_{1}, x_{2}, x_{3}\right) \mapsto\left(e^{2 \pi i x_{1}} e^{-2 \pi i x_{3}\left[x_{2}\right]}, x_{2}(\bmod 1), e^{2 \pi i x_{3}}\right),
$$

so, in $X$ we must have

$$
\lim _{c_{2} / 1}\left(w_{1}, c_{2}, w_{3}\right)=\left(w_{1} / w_{3}, 0, w_{3}\right)
$$

otherwise, the topology of $X=\mathbb{T} \times[0,1) \times \mathbb{T}$ is the product topology. We determine the action of $S$ on $X$ by looking at the image of

$$
\left(x_{1}, x_{2}, x_{3}\right)\left(w_{1}, c_{2}, w_{3}\right) \cong\left(x_{1}, x_{2}, x_{3}\right)\left(c_{1}, c_{2}, c_{3}\right)=\left(x_{1}+c_{1}+x_{3} c_{2}, x_{2}+c_{2}, x_{3}+c_{3}\right)
$$

in $X=\mathbb{T} \times[0,1) \times \mathbb{T}$. It is

$$
\left(e^{2 \pi i x_{1}} w_{1} e^{2 \pi i x_{3} c_{2}} e^{-2 \pi i x_{3}\left[x_{2}+c_{2}\right]} w_{3}^{-\left[x_{2}+c_{2}\right]},\left(x_{2}+c_{2}\right) \bmod 1, e^{2 \pi i x_{3}} w_{3}\right) .
$$

Thus we identify the transformation coming from $\left(x_{1}, x_{2}, x_{3}\right)$ with

$$
\begin{aligned}
& T=(\zeta, a, g, h) \in G:=\mathbb{T} \times[0,1) \times \mathbb{R}^{\mathcal{A P}} \times \mathbb{Z}^{\mathcal{A P}}, \\
& T=\left(e^{2 \pi i x_{1}} e^{-2 \pi i x_{3}\left[x_{2}\right]}, x_{2}(\bmod 1), e^{2 \pi i x_{3} \cdot},()^{-\left[x_{2}\right]}\right) ;
\end{aligned}
$$


then $T\left(w_{1}, c_{2}, w_{3}\right)=\left(\left(\zeta w_{1} g\left(c_{2}\right) h\left(w_{3}\right)\right) /\left(g(1) w_{3}\right),\left(a+c_{2}\right) \bmod 1, g(1) w_{3}\right)$, the denominator appearing if $a+c_{2} \geqslant 1$. So, in $G$ we must have

$$
\lim _{a / 1}(\zeta, a, g, h)=(\zeta / g(1), 0, g, h /())
$$

otherwise, the topology of $G=\mathbb{T} \times[0,1) \times \mathbb{R}^{\mathcal{A P}} \times \mathbb{Z}^{\mathcal{A P}}$ is the product topology. Writing out $T^{\prime} T\left(w_{1}, c_{2}, w_{3}\right)$, we find that

(*) $\quad T^{\prime} T=\left(\zeta^{\prime}, a^{\prime}, g^{\prime}, h^{\prime}\right)(\zeta, a, g, h)=\left(\frac{\zeta^{\prime} \zeta g^{\prime}(a) h^{\prime}(g(1))}{g^{\prime}(1) g(1)},\left(a^{\prime}+a\right) \bmod 1, g^{\prime} g, \frac{h^{\prime} h}{()}\right)$,

the denominators appearing if $a^{\prime}+a \geqslant 1$.

We continue our analysis of this example by observing that the map $\psi$, which sends $\left(x_{1}, x_{2}, x_{3}\right) \in S$ to $T=(\zeta, a, g, h) \in G$ (as above) has kernel $\mathbb{Z} \times\{0\} \times\{0\}$, a normal subgroup of $S$, and that $\psi(S)$ is dense in $G$, which is a group with inverse

$$
T^{-1}=(\zeta, a, g, h)^{-1}=\left(\frac{\zeta^{-1} g(a) h(g(1))}{g(1)}, 1-a, g^{-1}, h^{-1}()\right), 0<a<1,
$$

and $(\zeta, 0, g, h)^{-1}=\left(\zeta^{-1} h(g(1)), 0, g^{-1}, h^{-1}\right)$. The density claim follows from the last assertion of Theorem 4 , which says, in this setting, that the continuous characters on $\widehat{\mathbb{R}} \cong \mathbb{R},\left\{y \mapsto e^{2 \pi i x y} \mid x \in \mathbb{R}\right\}$, are dense in $\mathbb{R}^{\mathcal{A P}}$, and the continuous characters on $\widehat{\mathbb{Z}} \cong \mathbb{T},\left\{w \mapsto w^{n} \mid n \in \mathbb{Z}\right\}$, are dense in $\mathbb{Z}^{\mathcal{A P}}$. Thus

$$
\Lambda(G)=\mathbb{T} \times[0,1) \times \mathbb{R} \times \mathbb{Z} \subset \mathbb{T} \times[0,1) \times \mathbb{R}^{\mathcal{A P}} \times \mathbb{Z}^{\mathcal{A P}}=G
$$

We want to show that $L=\mathbb{T} \times\{0\} \times\{1\} \times\{1\}$, which also equals the centre $Z(G)$ of $G$. Now

$$
L=\bigcap\left\{U^{-1} U U^{-1} U \mid U \text { is a neighbourhood of the identity of } G\right\},
$$

that is, a member of $x \in L$ can be expressed in the form $x=T_{1}^{-1} T_{2} T_{3}^{-1} T_{4}$, where the $T$ 's can be arbitrarily near the identity of $G$. Bearing in mind that the topology and product for the last three coordinates are not quite what one would expect from the notation, one shows readily nonetheless that these coordinates of $x$ must be arbitrarily near the identity, and so must be the identity. Writing out the first coordinate of $T_{1}^{-1} T_{2} T_{3}^{-1} T_{4}$, one sees there are so many variables involved that there is plenty of room to use Kronecker's Theorem [6] and make this coordinate equal to any given $\xi \in \mathbb{T}$.

So, for $G=\mathbb{T} \times[0,1) \times \mathbb{R}^{\mathcal{A P}} \times \mathbb{Z}^{\mathcal{A P}}$, the compact topological groups of (*) are

$$
L=\mathbb{T} \times\{0\} \times\{1\} \times\{1\} \cong \mathbb{T} \text { and } G / L \cong[0,1) \times \mathbb{R}^{\mathcal{A P}} \times \mathbb{Z}^{\mathcal{A P}},
$$


the latter a compact, abelian, topological group, in which $\lim _{a / 1}(a, g, h)=(0, g, h /())$ and

$$
\left(a^{\prime}, g^{\prime}, h^{\prime}\right)(a, g, h)=\left(\left(a^{\prime}+a\right) \bmod 1, g^{\prime} g, h^{\prime} h /()\right),
$$

the denominator appearing in the last coordinate if $a^{\prime}+a \geqslant 1$. As was shown in Section 1 , Haar measure $\mu$ on $G$ is given for $f \in \mathcal{C}(G)$ by integrating with respect to $\mu_{1}$ and $\mu_{2}$, the Haar measures on the compact topological groups $L$ and $G / L$, respectively; for $f \in \mathcal{C}(G)$, put $\bar{f}(x)=\int_{L} L_{x} f d \mu_{1}$ and then $\mu(f)=\mu_{2}(f), \bar{f}$ being in $\mathcal{C}_{L}(G) \cong \mathcal{C}(G / L)$. Since $L \subset \Lambda(G) \cap Z(G)$, Theorem 2 shows that $\mu$ is uniquely determined by left invariance, as well as by right invariance. We must have $\mu_{2}\left(\{0\} \times \mathbb{R}^{\mathcal{A P}} \times \mathbb{Z}^{\mathcal{A P}}\right)=0$, so $\mu_{2}$ is still just the product of the Haar measures on (the compact topological groups) $[0,1), \mathbb{R}^{\mathcal{A P}}$ and $\mathbb{Z}^{\mathcal{A P}}$. Similarly, one sees that Haar measure $\mu$ on $G$ is just the product of the Haar measures on all four component groups on $G$.

The natural map

$$
\theta:(\zeta, a, g, h)=(\zeta, 0,1,1)(1, a, g, h) \mapsto(\zeta,(a, g, h)), G \rightarrow G^{\prime}:=L \times G / L
$$

is not a homeomorphism of $G$ onto the product of the compact spaces $L \cong \mathbb{T}$ and

$$
G / L \cong[0,1) \times \mathbb{R}^{\mathcal{A P}} \times \mathbb{Z}^{\mathcal{A P}},
$$

because $\{1\} \times[0,1) \times \mathbb{R}^{\mathcal{A P}} \times \mathbb{Z}^{\mathcal{A P}}$ is not a closed subset of $G$. However, $\theta$ is an algebraic isomorphism of $G$ onto $G^{\prime}$ with multiplication, as for $G$ in ( $\star$ ), given in Schreier formulation (1); the automorphisms of $L$ coming from $G / L$ are all just the identity (a situation we have called the "trivial homomorphism" case in [8]), and the function from $\left(t^{\prime}, t\right) \mapsto\left[t^{\prime}, t\right], G / L \times G / L \rightarrow L$ is given by

$$
\left(\left(a^{\prime}, g^{\prime}, h^{\prime}\right),(a, g, h)\right) \mapsto\left[\left(a^{\prime}, g^{\prime}, h^{\prime}\right),(a, g, h)\right]=\frac{g^{\prime}(a) h^{\prime}(g(1))}{g^{\prime}(1) g(1)} \in \mathbb{T},
$$

the denominator appearing if $a+a^{\prime} \geqslant 1$. This last function is not continuous in either variable, so $G^{\prime}$ is neither right topological nor left topological (analogously defined). The only difference in Schreier formulation (2) is that the order of the coordinates in $L \times G / L$ is reversed.

$G$ has another closed normal subgroup $G_{1}:=\mathbb{T} \times[0,1) \times\{1\} \times \mathbb{Z}^{\mathcal{A P}}$, whose quotient group $G / G_{1} \cong \mathbb{R}^{\mathcal{A P}}$ is (algebraically and topologically) isomorphic to $\{1\} \times$ $\{0\} \times \mathbb{R}^{\mathcal{A P}} \times\{1\} \subset G$. In Schreier formulation (1), the extension $G^{\prime \prime}:=G_{1} \times G / G_{1}$ has a somewhat simplified multiplication,

$$
\left(\left(\zeta^{\prime}, a^{\prime}, h^{\prime}\right), g^{\prime}\right)((\zeta, a, h), g)=\left(\left(\zeta^{\prime} \zeta g^{\prime}(a) h\left(g^{\prime-1}(1)\right),\left(a^{\prime}+a\right) \bmod 1, h^{\prime} h /()\right), g^{\prime} g\right),
$$

the donominator appearing if $a+a^{\prime} \geqslant 1$. However, the algebraic isomorphism

$$
(\zeta, a, g, h)=\left(\zeta h\left(g^{-1}(1), a, 1, h\right)(1,0, g, 1) \mapsto\left(\left(\zeta h\left(g^{-1}(1)\right), a, h\right), g\right), G \rightarrow G^{\prime \prime}\right)
$$

is not continuous, and $G^{\prime \prime}$ is neither right nor left topological. The situation is much the same in Schreier formulation (2). 
REMARKS. 1. Since the homomorphism $\psi: S=\mathbb{R}^{3} \rightarrow G$ has kernel $\mathbb{Z} \times\{0\} \times\{0\}$, we will end up with the same compact right topological group $G$ if we start with the Heisenberg group

$$
S^{\prime}=(S /(\mathbb{Z} \times\{0\} \times\{0\}) \cong) \mathbb{T} \times \mathbb{R} \times \mathbb{R}
$$

with multiplication

$$
\left(w^{\prime}, x_{1}^{\prime}, x_{2}^{\prime}\right)\left(w, x_{1}, x_{2}\right)=\left(w^{\prime} w e^{2 \pi i x_{2}^{\prime} x_{1}}, x_{1}^{\prime}+x_{1}, x_{2}^{\prime}+x_{2}\right)
$$

and subgroup $\{1\} \times \mathbb{Z} \times \mathbb{Z} \subset S^{\prime}$.

2. As we have stated, $\mathbb{R}^{3}$ (as above) is the (connected and simply connected) nilpotent Lie group of dimension 3. Nielsen [15] lists all such groups of dimension less than 7, along with much useful information about them. The methods here can be applied to these groups, with varying success.

(i) $S=G_{5,1}=\mathbb{R}^{5}$, with multiplication $\left(x_{1}^{\prime}, x_{2}^{\prime}, x_{3}^{\prime}, x_{4}^{\prime}, x_{5}^{\prime}\right)\left(x_{1}, x_{2}, x_{3}, x_{4}, x_{5}\right)$

$$
=\left(x_{1}^{\prime}+x_{1}+x_{3}^{\prime} x_{2}+x_{5}^{\prime} x_{4}, x_{2}^{\prime}+x_{2}, x_{3}^{\prime}+x_{3}, x_{4}^{\prime}+x_{4}, x_{5}^{\prime}+x_{5}\right)
$$

[15, p.4] and subgroup $H=\mathbb{Z}^{5}$. The compact right topological group coming from the distal flow $(S, S / H)$ may be identified as

$$
\begin{gathered}
G=\mathbb{T} \times[0,1) \times \mathbb{R}^{\mathcal{A P}} \times \mathbb{Z}^{\mathcal{A P}} \times[0,1) \times \mathbb{R}^{\mathcal{A P}} \times \mathbb{Z}^{\mathcal{A P}}, \\
x=\left(x_{1}, x_{2}, x_{3}, x_{4}, x_{5}\right) \in S \text { corresponding to } T=\left(\zeta, a_{1}, g_{1}, h_{1}, a_{2}, g_{2}, h_{2}\right)= \\
\left(e^{2 \pi i x_{1}} e^{-2 \pi i x_{3}\left[x_{2}\right]} e^{-2 \pi i x_{5}\left[x_{4}\right]}, x_{2}(\bmod 1), e^{2 \pi i x_{3} \cdot},()^{-\left[x_{2}\right]}, x_{4}(\bmod 1), e^{2 \pi i x_{5} \cdot},()^{-\left[x_{4}\right]}\right)
\end{gathered}
$$

in $G$, where

$$
\begin{aligned}
& \lim _{a_{1} / 1}\left(\zeta, a_{1}, g_{1}, h_{1}, a_{2}, g_{2}, h_{2}\right)=\left(\zeta / g_{1}(1), 0, g_{1}, h_{1} /(), a_{2}, g_{2}, h_{2}\right), \\
& \lim _{a_{2} \nearrow_{1}}\left(\zeta, a_{1}, g_{1}, h_{1}, a_{2}, g_{2}, h_{2}\right)=\left(\zeta / g_{2}(1), a_{1}, g_{1}, h_{1}, 0, g_{2}, h_{2} /()\right),
\end{aligned}
$$

and multiplication is given by

$$
\begin{gathered}
T^{\prime} T=\left(\zeta^{\prime} \zeta g_{1}^{\prime}\left(a_{1}\right) g_{2}^{\prime}\left(a_{2}\right) \frac{h_{1}^{\prime}\left(g_{1}(1)\right) h_{2}^{\prime}\left(g_{2}(1)\right)}{g_{1}^{\prime}(1) g_{1}(1) g_{2}^{\prime}(1) g_{2}(1)},\right. \\
\left.\left(a_{1}^{\prime}+a_{1}\right) \bmod 1, g_{1}^{\prime} g_{1}, \frac{h_{1}^{\prime} h_{1}}{()},\left(a_{2}^{\prime}+a_{2}\right) \bmod 1, g_{2}^{\prime} g_{2}, \frac{h_{2}^{\prime} h_{2}}{()}\right),
\end{gathered}
$$

where the denominators involving subscript $j$ appear if $a_{j}^{\prime}+a_{j} \geqslant 1, j=1,2$. Again, the image of $S$ is dense in $G$. Also

$$
\Lambda(G)=\mathbb{T} \times[0,1) \times \mathbb{R} \times \mathbb{Z} \times[0,1) \times \mathbb{R} \times \mathbb{Z} \subset G
$$


and $L=Z(G)=\mathbb{T} \times\{0\} \times\{1\} \times\{1\} \times\{0\} \times\{1\} \times\{1\}$, so Haar measure $\mu$, which is again just the product of the Haar measures on the component groups, is uniquely determined by left invariance, as well as by right invariance.

(ii) $S=G_{5,2}=\mathbb{R}^{5}$ with multiplication $\left(x_{1}^{\prime}, x_{2}^{\prime}, x_{3}^{\prime}, x_{4}^{\prime}, x_{5}^{\prime}\right)\left(x_{1}, x_{2}, x_{3}, x_{4}, x_{5}\right)$

$$
=\left(x_{1}^{\prime}+x_{1}+x_{5}^{\prime} x_{3}, x_{2}^{\prime}+x_{2}+x_{5}^{\prime} x_{4}, x_{3}^{\prime}+x_{3}, x_{4}^{\prime}+x_{4}, x_{5}^{\prime}+x_{5}\right)
$$

$\left[15\right.$, p.6] and subgroup $H=\mathbb{Z}^{5}$. Much as for $G_{5,1}$ in (i), here $X=S / H$ and the compact right topological group $G=S^{-} \subset X^{X}$ turns out to be

$$
\mathbb{T} \times[0,1) \times \mathbb{R}^{\mathcal{A P}} \times \mathbb{Z}^{\mathcal{A P}} \times \mathbb{T} \times[0,1) \times \mathbb{Z}^{\mathcal{A P}}
$$

with multiplication

$$
\begin{aligned}
T^{\prime} T= & \left(\zeta_{1}^{\prime}, a_{1}^{\prime}, g^{\prime}, h_{1}^{\prime}, \zeta_{2}^{\prime}, a_{2}^{\prime}, h_{2}^{\prime}\right)\left(\zeta_{1}, a_{1}, g, h_{1}, \zeta_{2}, a_{2}, h_{2}\right) \\
= & \left(\zeta_{1}^{\prime} \zeta_{1} \frac{g^{\prime}\left(a_{1}\right) h_{1}^{\prime}(g(1))}{g^{\prime}(1) g(1)},\left(a_{1}^{\prime}+a_{1}\right) \bmod 1, g^{\prime} g, \frac{h_{1}^{\prime} h_{1}}{()},\right. \\
& \left.\zeta_{2}^{\prime} \zeta_{2} \frac{g^{\prime}\left(a_{2}\right) h_{2}^{\prime}(g(1))}{g^{\prime}(1) g(1)},\left(a_{2}^{\prime}+a_{2}\right) \bmod 1, \frac{h_{2}^{\prime} h_{2}}{()}\right),
\end{aligned}
$$

where the denominators in terms involving subscript $j$ appear if $a_{j}^{\prime}+a_{j} \geqslant 1, j=1,2$. The interested reader can fill in the other details.

(iii) $S=G_{6,15}=\mathbb{R}^{6}$, with multiplication

$$
\begin{gathered}
\left(x_{1}^{\prime}, x_{2}^{\prime}, x_{3}^{\prime}, x_{4}^{\prime}, x_{5}^{\prime}, x_{6}^{\prime}\right)\left(x_{1}, x_{2}, x_{3}, x_{4}, x_{5}, x_{6}\right) \\
=\left(x_{1}^{\prime}+x_{1}+x_{6}^{\prime} x_{4}, x_{2}^{\prime}+x_{2}+x_{5}^{\prime} x_{4}, x_{3}^{\prime}+x_{3}+x_{6}^{\prime} x_{5}, x_{4}^{\prime}+x_{4}, x_{5}^{\prime}+x_{5}, x_{6}^{\prime}+x_{6}\right)
\end{gathered}
$$

[15, p.78] and subgroup $H=\mathbb{Z}^{6}$, can also be dealt with successfully, because the multiplication formula has only quadratic terms. However, ...

(iv) $S=G_{4}=\mathbb{R}^{4}$ with multiplication $\left(x_{1}^{\prime}, x_{2}^{\prime}, x_{3}^{\prime}, x_{4}^{\prime}\right)\left(x_{1}, x_{2}, x_{3}, x_{4}\right)$

$$
=\left(x_{1}^{\prime}+x_{1}+\left(x_{4}^{\prime 2} x_{3}\right) / 2, x_{2}^{\prime}+x_{2}+x_{4}^{\prime} x_{3}, x_{3}^{\prime}+x_{3}, x_{4}^{\prime}+x_{4}\right)
$$

$[15, \mathrm{p} .2]$ and subgroup $H=\mathbb{Z} / 2 \times \mathbb{Z}^{2}$. The quotient space $X=S / H$ can be identified with $\mathbb{T}^{4}$ in a satisfactory way, but the cubic term in the multiplication formula presents difficulties in the identification of $S^{-} \subset X^{X}$ that we have not overcome to our satisfaction.

EXAMPLE 2. Let $G^{\prime}=\mathbb{T} \times \mathbb{T}^{\mathbb{T}}$, where $\mathbb{T}$ is the circle group, and multiplication is given by $\left(w^{\prime \prime}, h^{\prime \prime}\right)\left(w^{\prime}, h^{\prime}\right)=\left(w^{\prime \prime} w^{\prime}, R_{w^{\prime}} h^{\prime \prime} h^{\prime}\right)$. (We are using Schreier formulation (2) here, and are just using the right translation notation $R_{w^{\prime}} h^{\prime \prime}$ for functions $h^{\prime \prime} \in \mathbb{T}^{\mathbb{T}}$, instead 
of $\left(h^{\prime \prime}\right) w^{\prime}=\left(h^{\prime \prime}\right)_{w^{\prime}} \mathcal{R}$ as in Section 1.) $G^{\prime}$ is a compact right topological group; it arises when we consider the Euclidean group of the plane $S=\mathbb{T} \times \mathbb{C}$ with multiplication

$$
\left(w^{\prime}, z^{\prime}\right)(w, z)=\left(w^{\prime} w, z^{\prime} w+z\right)
$$

(also in Schreier formulation (2)) and its subgroup

$$
H=\{1\} \times(\mathbb{Z}+i \mathbb{Z})\left(\cong \mathbb{Z}^{2}\right) \subset\{1\} \times \mathbb{C} .
$$

The quotient space $X=S / H$ is identified with $\mathbb{T}^{3}$ via

$$
(w, z) H=(w, z+H) \mapsto(w, v, u):=\left(w, e^{2 \pi i R_{e} z}, e^{2 \pi i \operatorname{Im} z}\right) .
$$

Inspection of $\left(w^{\prime}, z^{\prime}\right)(w, z) H$ shows that the appropriate action of $S$ on $X$ is

$$
\begin{gathered}
\left(w^{\prime}, z^{\prime}\right):(w, v, u) \mapsto\left(w^{\prime} w, e^{2 \pi i \operatorname{Re}\left(z^{\prime} w\right)} v, e^{2 \pi i \operatorname{Im}\left(z^{\prime} w\right)} u\right) \\
=\left(w^{\prime} w, h^{\prime}(w) v, h^{\prime}(w / i) u\right)
\end{gathered}
$$

if we write $h^{\prime}(w)=e^{2 \pi i \mathcal{R} e\left(z^{\prime} w\right)}=e^{2 \pi i\left|z^{\prime}\right| \cos \left(\operatorname{Arg}\left(z^{\prime} w\right)\right)}$. Thus, the transformation

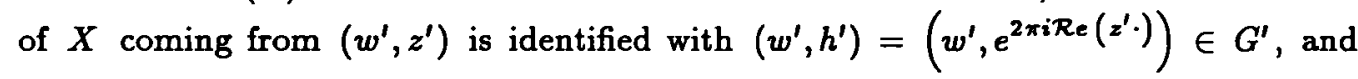
the transformation of $X$ coming from $\left(w^{\prime \prime}, z^{\prime \prime}\right)\left(w^{\prime}, z^{\prime}\right)=\left(w^{\prime \prime} w^{\prime}, z^{\prime \prime} w^{\prime}+z^{\prime}\right)$ is identified with

$$
\left(w^{\prime \prime} w^{\prime}, R_{w^{\prime}} h^{\prime \prime} h^{\prime}\right)=\left(w^{\prime \prime}, h^{\prime \prime}\right)\left(w^{\prime}, h^{\prime}\right) \in G^{\prime} .
$$

(The flow $(S, X)$ was arrived at by a different route in [8].)

As noted in [8], the closure $G:=S^{-} \subset G^{\prime}$ is a lot of $G^{\prime}$, but not all of it. It is clear that $G=\mathbb{T} \times K$ for a closed translation invariant subgroup $K$ of $\mathbb{T}^{\mathbb{T}} ; h^{\prime} \in K$ if and only if there is a net $\left\{z_{\alpha}\right\} \subset \mathbb{C}$ such that

$$
h_{\alpha}:=e^{2 \pi i R_{e}\left(z_{\alpha} \cdot\right)}=e^{2 \pi i\left|z_{\alpha}\right| \cos \left(\operatorname{Arg}\left(z_{\alpha} \cdot\right)\right)} \rightarrow h^{\prime}
$$

pointwise on $\mathbb{T}$. Thus there is a natural injection $f \mapsto h_{f}, \mathbb{R}^{\mathcal{A P}} \rightarrow K$ given by $h_{f}(w)=f(\mathcal{R} e w)$. But $K$ must be much larger than this image of $\mathbb{R}^{\mathcal{A P}}$ because of the possibility of varying $\operatorname{Arg}\left(z_{\alpha}\right)$, as well as $\left|z_{\alpha}\right|$, in (3). On the other hand, trigonometric identities show that there are many restrictions on the $h^{\prime}$ 's in $K$. In particular, we have for all $\theta, \psi, \eta \in \mathbb{R}$ and $m=2,3,4, \ldots$

(i) $\cos (\theta+\psi)=-\cos (\theta+\pi+\psi)$, and more generally

(ii) $\Sigma_{k=0}^{m-1} \cos (\theta+2 \pi k / m+\psi)=0$; and

(iii) $2 \cos \eta \cos (\theta+\psi)=\cos (\theta+\eta+\psi)+\cos (\theta-\eta+\psi)$.

These imply that, for any $h^{\prime}=e^{2 \pi i R e\left(z^{\prime} \cdot\right)}, z^{\prime} \in \mathbb{C}$, and hence for any $h^{\prime} \in K$, we must have for all $w \in \mathbb{T}$ and $m=2,3,4, \ldots$

(i) $h^{\prime}(-w)=\overline{h^{\prime}(w)}$;

(ii) $\Pi_{k=0}^{m-1} h^{\prime}\left(w e^{2 \pi i k / m}\right)=1$; and

(iii) $h^{\prime}(w)^{2 p}=h^{\prime}\left(w e^{i \eta}\right)^{q} h^{\prime}\left(w e^{-i \eta}\right)^{q}$, if $\cos \eta=p / q \in \mathbb{Q}$. 
Beyond these comments, we are unable to describe the subgroup $K$ of $\mathbb{T}^{\mathbb{T}}$ explicitly.

We give some additional information about $G$. (The corresponding information about $G^{\prime}$ is easier, sometimes trivial, to arrive at.) For a start, the centre $Z(G)=\{e\}$ and the topological centre of $G$,

$$
\Lambda(G)=\left\{\left(w^{\prime}, h^{\prime}\right) \mid h^{\prime} \in K \text { is continuous }\right\} .
$$

It seems very likely that the subgroup $L$ of $G$ is $\{1\} \times K$; however, we are not able to prove that this is the case. Nonetheless, we can determine Haar measure $\mu$ on $G$, with the method of Section 1, from the Haar measures on the compact topological groups $\{1\} \times K \cong K$ and $G /(\{1\} \times K) \cong \mathbb{T} ;$ in fact, $\mu$ is just the product of the Haar measures on $\mathbb{T}$ and $K$ (which one verifies readily to be right invariant). We do know that $L$ is not trivial, and expect that the continuity hypothesis of Corollary 3 for Schreier formulation (2) is not satisfied. (It is not satisfied if $L=K$.) So, we doubt that this corollary (or Theorem 2) can be used to conclude that $\mu$ is the unique left invariant probability measure on $G$; perhaps it is not!

Instead of $H=\{1\} \times(\mathbb{Z}+i \mathbb{Z}) \subset S=\mathbb{T} \times \mathbb{C}$, one might take $n=3,4$, or 6 and root of unity $\alpha=e^{2 \pi i / n}$, and start with subgroup

$$
H^{\prime}:=\left\{\alpha^{k} \mid 0 \leqslant k<n\right\} \times\{k+m \alpha \mid k, m \in \mathbb{Z}\} \subset S=\mathbb{T} \times \mathbb{C} .
$$

The Ellis group of such a distal flow $\left(S, G / H^{\prime}\right)$ is not much different from that of $G / H$, a little more complicated topologically.

Example 3. Let $G=\mathbb{T} \times E$, where $\mathbb{T}$ is the circle group and $E=E(\mathbb{T})$ is the set of all endomorphisms (continuous or not) of $\mathbb{T}$; as indicated in Theorem 4,E may be identified with the almost periodic compactification of $\mathbb{Z}, E=\left(\mathbb{T}_{d}\right)^{-}=\left((\widehat{\mathbb{Z}})_{d}\right)^{-}=$ $\mathbb{Z}^{\mathcal{A P}}$, where $\mathbb{T}_{d}$ denotes $\mathbb{T}$ with the discrete topology. The operation

$$
\left(w^{\prime}, h^{\prime}\right)(w, h)=\left(w^{\prime} w h^{\prime} \circ h\left(e^{2 i}\right), h^{\prime} h\right)
$$

makes $G$ a compact right topological group with Schreier multiplication (1); the automorphisms $w \mapsto h(w)$ of $\mathbb{T}$ are all trivial, that is, the identity, and the function

$$
\left(h^{\prime}, h\right) \mapsto\left[h^{\prime}, h\right], G_{2} \times G_{2} \rightarrow G_{1}
$$

is given by $\left[h^{\prime}, h\right]=h^{\prime} \circ h\left(e^{2 i}\right)$ (which is continuous only in the variable $h^{\prime}$ ). $G$ is the Ellis group of the distal flow $\left(\mathbb{Z}, \mathbb{T}^{2}\right)$, which has action

$$
n:\left(w_{1}, w_{2}\right) \mapsto\left(e^{2 n i} w_{1}, e^{n^{2} i} w_{1}^{n} w_{2}\right)
$$


namely, $n \in \mathbb{Z}$ corresponds to $\left(e^{n^{2} i},()^{n}\right) \in G$ (where ()$^{n}: v \mapsto v^{n}$ ), and the resulting isomorphic image of $\mathbb{Z}$ is dense in $G$. The topological centre of $G, \Lambda(G)$, equals

$$
\{(w, h) \in G \mid h \in E \text { is continuous }\}=\left\{\left(w,()^{n}\right) \in G \mid n \in \mathbb{Z}\right\}
$$

See [14] for the claims of the last two sentences, especially for the proof that $\mathbb{Z}^{-}=G$.

So, for $G=\mathbb{T} \times E$, the compact topological groups of $(*)$ are $L=\mathbb{T} \times\{e\} \cong \mathbb{T}$ and $G / L \cong E$. As for Example 2, Haar measure $\mu$ on $G$ is just the product of the Haar measures on $\mathbb{T}$ and $E$. Furthermore, since $G=\mathbb{T} \times E=G_{1} \times G_{2}$ has multiplication in Schreier formulation (1), the corollary tells us that $\mu$ is uniquely determined by left invariance. At first glance, it seems strange that there are much larger compact, normal, topological, even abelian subgroups $L^{\prime}$ of $G$ with $G / L^{\prime}$ a compact topological group. For example, if $E_{1}:=\left\{h \in E \mid h\left(e^{2 i}\right)=1\right\}$, then $L^{\prime}:=\mathbb{T} \times E_{1}$ is such a subgroup, with quotient $G / L^{\prime}$ isomorphic to $\mathrm{T}$ via the quotient map $(w, h) \mapsto h\left(e^{2 i}\right)$. Similarly,

$$
L^{\prime \prime}:=\mathbb{T} \times\left\{h \in E \mid h\left(e^{2 i}\right)=1=h\left(e^{\sqrt{2} i}\right)\right\}
$$

is another such subgroup of $G$; now $G / L^{\prime \prime}$ is isomorphic to $\mathbb{T}^{2}$.

The normal subgroup $L^{\prime}$ of $G$ permits us to consider $G$ as an extension of $L^{\prime}$ by $G / L^{\prime} \cong \mathbb{T}$. Insofar as we have been able to write out the details, it seems that the product in $L^{\prime} \times \mathbb{T}$ (in either Schreier formulation) and the algebraic isomorphism $G \rightarrow L^{\prime} \times \mathbb{T}$ must be messy algebraically, and very bad topologically; in particular, $G$ is not homeomorphic to the product space $L^{\prime} \times \mathbb{T}$. However, the method of Section 1 can still be used to express Haar measure $\mu$ on $G$ in terms of the Haar measures on the compact topological groups $L^{\prime}$ and $\mathbb{T}$.

\section{REFERENCES}

[1] H. Anzai and S. Kakutani, 'Bohr compactifications of a locally compact abelian group I, II', Proc. Imp. Acad. Tokyo 19 (1943), 476-480, 533-539.

[2] L. Auslander and F. Hahn, 'Real functions coming from flows on compact spaces and concepts of almost periodicity', Trans. Amer. Math. Soc. 106 (1963), 415-426.

[3] J.F. Berglund, H.D. Junghenn and P. Milnes, Analysis on semigroups: function spaces, compactifactions, representations (Wiley, New York, 1989).

[4] R. Ellis, 'Distal transformation groups', Pacific J. Math. 9 (1958), 401-405.

[5] R. Ellis, Lectures on topological dynamics (Benjamin, New York, 1969).

[6] E. Hewitt and K.A. Ross, Abstract harmonic analysis I (Springer-Verlag, Berlin, Heidelberg, New York, 1963).

[7] A.W. Knapp, 'Distal functions on groups', Trans. Amer. Math. Soc. 128 (1967), 1-40.

[8] P. Milnes, 'Ellis groups and group extensions', Houston J. Math. 12 (1986), 87-108. 
[9] P. Milnes, 'Enveloping semigroups, distal flows and groups of Heisenberg type', Houston J. Math. 15 (1989), 563-572.

[10] P. Milnes, 'Extension of flows, Ellis groups and groups of Heisenberg type', Math. Nach. 150 (1991), 97-108.

[11] P. Milnes and J. Pym, 'Haar measure for compact right topological groups', Proc. Amer. Math. Soc. (to appear).

[12] P. Milnes and J. Pym, 'Homomorphisms of minimal and distal flows', (submitted).

[13] I. Namioka, 'Right topological groups, distal flows and a fixed point theorem', Math. Systems Theory 6 (1972), 193-209.

[14] I. Namioka, 'Ellis groups and compact right topological groups', in Contemporary Mathematics 26: Conference in modern analysis and probability, pp. 295-300 (Amer. Math. Soc., 1984).

[15] O.A. Nielsen, Unitary representations and coadjoint orbits of low-dimensional nilpotent lie groups (Queen's Papers in Pure and Applied Mathematics, no. 63, Kingston, Ont., 1983).

[16] W.A.F. Ruppert, 'Über kompakte rechtstopologische Gruppen mit gleichgradig stetigen Linkstranslationen', Sitzungsber. Österreichischen Akad. Wiss. Math.-Natur. Kl. 184 (1975), 159-169.

[17] G. Zappa, 'Sulla construzione dei gruppi di due dati sottogruppi permutabili tra loro', Atti Secundo Congresso Unione Matematica Italiana, Bologna (1940), 119-125.

Department of Mathematics

University of Western Ontario

London

Ontario N6A 5 B7

Canada 